\title{
BOUNDING THE CONVEX COMBINATION OF ARITHMETIC AND INTEGRAL MEANS IN TERMS OF ONE-PARAMETER HARMONIC AND GEOMETRIC MEANS
}

\author{
WEI-MAO QIAN, WEN ZHANG, AND YU-MING CHU
}

Received 22 May, 2017

Abstract. In the article, we find the best possible parameters $\lambda_{1}, \mu_{1}, \lambda_{2}$ and $\mu_{2}$ on the interval $[0,1 / 2]$ such that the double inequalities

$$
\begin{gathered}
H\left(a, b ; \lambda_{1}\right)<\alpha A(a, b)+(1-\alpha) T(a, b)<H\left(a, b ; \mu_{1}\right), \\
G\left(a, b ; \lambda_{2}\right)<\alpha A(a, b)+(1-\alpha) T(a, b)<G\left(a, b ; \mu_{2}\right)
\end{gathered}
$$

hold for all $\alpha \in[0,1]$ and $a, b>0$ with $a \neq b$, where $A(a, b)=(a+b) / 2, T(a, b)=$ $2 \int_{0}^{\pi / 2} a^{\cos ^{2} \theta} b^{\sin ^{2} \theta} d \theta / \pi, H(a, b ; \lambda)=2[\lambda a+(1-\lambda) b][\lambda b+(1-\lambda) a] /(a+b), G(a, b ; \mu)=$ $\sqrt{[\mu a+(1-\mu) b][\mu b+(1-\mu) a]}$ are the arithmetic, integral, one-parameter harmonic and oneparameter geometric means of $a$ and $b$, respectively.

2010 Mathematics Subject Classification: 26E60; 33C10

Keywords: integral mean, modified Bessel function, arithmetic mean, harmonic mean, geometric mean

\section{INTRODUCTION}

For $\lambda, \mu \in[0,1]$, the arithmetic mean $A(a, b)$, harmonic mean $H(a, b)$, geometric mean $G(a, b)$, integral mean $T(a, b)$ [25], one-parameter harmonic mean $H(a, b ; \lambda)$ and one-parameter geometric mean $G(a, b ; \mu)$ of two distinct positive real numbers $a$ and $b$ are given by

$$
\begin{gathered}
A(a, b)=\frac{a+b}{2}, \quad H(a, b)=\frac{2 a b}{a+b}, \\
G(a, b)=\sqrt{a b}, \quad T(a, b)=\frac{2}{\pi} \int_{0}^{\pi / 2} a^{\cos ^{2} \theta} b^{\sin ^{2} \theta} d \theta, \\
H(a, b ; \lambda)=H[\lambda a+(1-\lambda) b, \lambda b+(1-\lambda) b], \\
G(a, b ; \mu)=G[\mu a+(1-\mu) b, \mu b+(1-\mu) b],
\end{gathered}
$$

The first author was supported in part by the Natural Science Fund, Grant No. 2018 YZ07. 
respectively. The integral mean $T(a, b)$ has been the subject of intensive research in recent years due to it has been widely applied in pure and applied mathematics, physics and other natural sciences $[2,4-12,14-16,19,21,22,24,26-30,36-38,40]$.

The identity

and inequalities

$$
T(a, b)=\sqrt{a b} I_{0}(\log \sqrt{b / a})
$$

$$
L(a, b)<T(a, b)<\frac{A(a, b)+G(a, b)}{2}<\frac{2 A(a, b)+G(a, b)}{3}<I(a, b)
$$

for all $a, b>0$ with $a \neq b$ were established by Qi, Shi, Liu and Yang [18], where

$$
I_{\nu}(t)=\sum_{n=0}^{\infty} \frac{1}{n ! \Gamma(n+v+1)}\left(\frac{t}{2}\right)^{2 n+v}
$$

is the modified Bessel function of the first kind [1], $\Gamma(x)=\int_{0}^{\infty} e^{-t} t^{x-1} d t$ is the classical gamma function [13,39], and $L(a, b)=(b-a) /(\log b-\log a)$ and $I(a, b)=$ $\left(b^{b} / a^{a}\right)^{1 /(b-a)} / e$ are respectively the logarithmic and inentric means of $a$ and $b$.

Yang and Chu [31,32], and Yang, Chu and Song [33] proved that the inequalities

$$
\begin{aligned}
& \lambda_{1} \sqrt{L(a, b) A(a, b)}<T(a, b)<\mu_{1} \sqrt{L(a, b) A(a, b)}, \\
& L^{\lambda_{2}}(a, b) A^{1-\lambda_{2}}(a, b)<T(a, b)<\mu_{2} L(a, b)+\left(1-\mu_{2}\right) A(a, b), \\
& T(a, b)>L_{p}(a, b) \\
& \lambda_{3} \sqrt{L(a, b) I(a, b)}<T(a, b)<\mu_{3} \sqrt{L(a, b) I(a, b)},
\end{aligned}
$$

hold for all $a, b>0$ with $a \neq b$ if and only if $\lambda_{1} \leq \sqrt{2 / \pi}, \mu_{1} \geq 1, \lambda_{2} \geq 3 / 4, \mu_{2} \leq$ $3 / 4, p \leq 3 / 2, \lambda_{3} \leq \sqrt{e / \pi}$ and $\mu_{3} \geq 1$, where $L_{p}(a, b)=\left[\left(b^{p}-a^{p}\right) /(p(\log b-\right.$ $\log a))]^{1 / p}$ is the $p$-order generalized logarithmic mean of $a$ and $b$.

In [20], the authors proved that $p_{1}=0, q_{1}=1 / 4, p_{2}=0$ and $q_{2}=1 / 2-\sqrt{2} / 4$ are the best possible parameters on the interval $[0,1 / 2]$ such that the double inequalities

$$
\begin{gathered}
H\left(a, b ; p_{1}\right)<T(a, b)<H\left(a, b ; q_{1}\right), \\
G\left(a, b ; p_{2}\right)<T(a, b)<G\left(a, b ; q_{2}\right)
\end{gathered}
$$

hold for all $a, b>0$ with $a \neq b$.

Let $\alpha \in[0,1], x \in[0,1 / 2], a, b>0$ with $a \neq b, f(x)=H(a, b ; x), g(x)=$ $G(a, b ; x)$. Then we clearly see that both the functions $f(x)$ and $g(x)$ are strictly increasing on $[0,1 / 2]$. Inequality (1.6) and the well known inequalities

$$
H(a, b)<G(a, b)<L(a, b)<I(a, b)<A(a, b)
$$

lead to the conclusion that

$$
\begin{aligned}
& f(0)=H(a, b)<\alpha A(a, b)+(1-\alpha) T(a, b)<A(a, b)=f(1 / 2), \\
& g(0)=G(a, b)<\alpha A(a, b)+(1-\alpha) T(a, b)<A(a, b)=g(1 / 2) .
\end{aligned}
$$


From inequalities (1.10) and (1.11) together with the monotonicity of the functions $f(x)$ and $g(x)$ on the interval $[0,1 / 2]$, it is necessary to discover the best possible parameters $\lambda_{1}, \mu_{1}, \lambda_{2}$ and $\mu_{2}$ on the interval $[0,1 / 2]$ such that the double inequalities

$$
\begin{gathered}
H\left(a, b ; \lambda_{1}\right)<\alpha A(a, b)+(1-\alpha) T(a, b)<H\left(a, b ; \mu_{1}\right), \\
G\left(a, b ; \lambda_{2}\right)<\alpha A(a, b)+(1-\alpha) T(a, b)<G\left(a, b ; \mu_{2}\right)
\end{gathered}
$$

hold for all $\alpha \in[0,1]$ and $a, b>0$ with $a \neq b$.

\section{LEMMAS}

Lemma 1 (Theorem 2.18 in [3]). The identity

$$
\Gamma\left(n+\frac{1}{2}\right)=\frac{(2 n) !}{2^{2 n} n !} \sqrt{\pi}
$$

holds for all $n \in \mathbb{N}$.

Lemma 2 ([17]). Let $\left\{a_{n}\right\}_{n=0}^{\infty}$ and $\left\{b_{n}\right\}_{n=0}^{\infty}$ be two real sequences with $b_{n}>0$ and $\lim _{n \rightarrow \infty} a_{n} / b_{n}=s$. Then the power series $\sum_{n=0}^{\infty} a_{n} t^{n}$ is convergent for all $t \in \mathbb{R}$ and

$$
\lim _{t \rightarrow \infty} \frac{\sum_{n=0}^{\infty} a_{n} t^{n}}{\sum_{n=0}^{\infty} b_{n} t^{n}}=s
$$

if the power series $\sum_{n=0}^{\infty} b_{n} t^{n}$ is convergent for all $t \in \mathbb{R}$.

Lemma 3 (Lemma 2.2 in [35]). The double inequality

$$
\frac{1}{(x+a)^{1-a}}<\frac{\Gamma(x+a)}{\Gamma(x+1)}<\frac{1}{x^{1-a}}
$$

holds for all $x>0$ and $a \in(0,1)$.

Lemma 4 ([34]). Let $A(t)=\sum_{k=0}^{\infty} a_{k} t^{k}$ and $B(t)=\sum_{k=0}^{\infty} b_{k} t^{k}$ be two real power series converging on $(-r, r)(r>0)$ with $b_{k}>0$ for all $k$. If the non-constant sequence $\left\{a_{k} / b_{k}\right\}_{k=0}^{\infty}$ is increasing (decreasing) for all $k$, then the function $t \mapsto$ $A(t) / B(t)$ is strictly increasing (decreasing) on $(0, r)$.

Lemma 5 ((3.5) in [23]). The identity

$$
I_{\lambda}(t) I_{\mu}(t)=\sum_{n=0}^{\infty} \frac{\Gamma(2 n+\lambda+\mu+1)}{n ! \Gamma(n+\lambda+\mu+1) \Gamma(n+\lambda+1) \Gamma(n+\mu+1)}\left(\frac{t}{2}\right)^{2 n+\lambda+\mu}
$$

holds for all $\lambda, \mu>-1$ and $t \in \mathbb{R}$.

Lemma 6. The identity

$$
\cosh (t) I_{0}(t)=\sum_{n=0}^{\infty} \frac{(4 n) !}{2^{2 n}[(2 n) !]^{3}} t^{2 n}
$$

holds for all $t \in \mathbb{R}$, where $\cosh (t)=\left(e^{t}+e^{-t}\right) / 2$ is the hyperbolic cosine functions. 
Proof. It follows from (1.7) and Lemmas 1 and 5 that

$$
\begin{aligned}
I_{-1 / 2}(t) & =\sqrt{\frac{2}{\pi t}} \sum_{n=0}^{\infty} \frac{t^{2 n}}{(2 n) !}=\sqrt{\frac{2}{\pi t}} \cosh (t), \\
\cosh (t) I_{0}(t) & =\sqrt{\frac{\pi t}{2}} I_{-1 / 2}(t) I_{0}(t) \\
& =\sqrt{\frac{\pi t}{2}} \sum_{n=0}^{\infty} \frac{\Gamma\left(2 n+\frac{1}{2}\right)}{\left[n ! \Gamma\left(n+\frac{1}{2}\right)\right]^{2}}\left(\frac{t}{2}\right)^{2 n-1 / 2}=\sum_{n=0}^{\infty} \frac{(4 n) !}{2^{2 n}[(2 n) !]^{3}} t^{2 n} .
\end{aligned}
$$

Lemma 7. The function

$$
f(t)=\frac{\cosh ^{2}(t)-\cosh (t) I_{0}(t)}{\sinh ^{2}(t)}
$$

is strictly increasing from $(0, \infty)$ onto $(1 / 4,1)$, where $\sinh (t)=\left(e^{t}-e^{-t}\right) / 2$ is the hyperbolic sine function.

Proof. Let $n \in \mathbb{N}$, and $\left\{a_{n}\right\}$ and $\left\{b_{n}\right\}$ be defined by

$$
a_{n}=\frac{(4 n+4) !}{2^{2 n+2}[(2 n+2) !]^{3}}, \quad b_{n}=\frac{2^{2 n+2}}{(2 n+2) !},
$$

respectively.

Then simple computations lead to

$$
\begin{gathered}
\frac{a_{0}}{b_{0}}=\frac{3}{8}, \\
\frac{a_{n}}{b_{n}}=\frac{(4 n+4) !}{2^{4 n+4}[(2 n+2) !]^{2}}, \\
\frac{a_{n+1}}{b_{n+1}}-\frac{a_{n}}{b_{n}}=-\frac{(n+2)(2 n+3)(8 n+13)(4 n+4) !}{2^{4 n+5}[(2 n+4) !]^{2}}<0
\end{gathered}
$$

for all $n \in \mathbb{N}$.

It follows from Lemmas 1, 2, 3 and 6 together with (2.1)-(2.4) that

$$
\begin{aligned}
f(t) & =1-\frac{2\left[\cosh (t) I_{0}(t)-1\right]}{\cosh (2 t)-1} \\
& =1-\frac{2 \sum_{n=1}^{\infty} \frac{(4 n) !}{2^{2 n}[(2 n) !]^{3}} t^{2 n}}{\sum_{n=1}^{\infty} \frac{2^{2 n}}{(2 n) !} t^{2 n}}=1-\frac{2 \sum_{n=0}^{\infty} a_{n} t^{2 n}}{\sum_{n=0}^{\infty} b_{n} t^{2 n}}, \\
f\left(0^{+}\right) & =1-\frac{2 a_{0}}{b_{0}}=\frac{1}{4},
\end{aligned}
$$




$$
\begin{aligned}
\frac{1}{\sqrt{\pi(2 n+5 / 2)}}<\frac{a_{n}}{b_{n}}=\frac{\Gamma(2 n+5 / 2)}{\sqrt{\pi} \Gamma(2 n+3)}<\frac{1}{\sqrt{\pi(2 n+2)}}, \\
f(\infty)=1-2 \lim _{n \rightarrow \infty} \frac{a_{n}}{b_{n}}=1 .
\end{aligned}
$$

From Lemma 4, (2.5) and (2.6) we clearly see that the function $f(t)$ is strictly increasing on $(0, \infty)$. Therefore, Lemma 7 follows from (2.7) and (2.8) together with the monotonicity of $f(t)$ on the interval $(0, \infty)$.

Lemma 8. The function

$$
g(t)=\frac{\cosh ^{2}(t)-I_{0}^{2}(t)}{\sinh ^{2}(t)}
$$

is strictly increasing from $(0, \infty)$ onto $(1 / 2,1)$.

Proof. Let $n \in \mathbb{N}$, and $\left\{c_{n}\right\}$ and $\left\{d_{n}\right\}$ be defined by

$$
c_{n}=\frac{(2 n+2) !}{2^{2 n+2}[(n+1) !]^{4}}, \quad d_{n}=\frac{2^{2 n+2}}{(2 n+2) !},
$$

respectively. Then simple computations lead to

$$
\begin{gathered}
\frac{c_{0}}{d_{0}}=\frac{1}{4}, \\
\frac{c_{n}}{d_{n}}=\frac{[(2 n+2) !]^{2}}{2^{4 n+4}[(n+1) !]^{4}}, \\
\frac{c_{n+1}}{d_{n+1}}-\frac{c_{n}}{d_{n}}=-\frac{(4 n+7)(n+2)^{2}[(2 n+2) !]^{2}}{2^{4 n+6}[(n+1) !]^{4}}<0
\end{gathered}
$$

for all $n \in \mathbb{N}$.

From Lemmas 1, 2, 3 and 5 together with (2.9)-(2.12) one has

$$
\begin{aligned}
I_{0}^{2}(t) & =\sum_{n=0}^{\infty} \frac{(2 n) !}{2^{2 n}(n !)^{4}} t^{2 n}, \\
g(t) & =1-\frac{2\left[I_{0}^{2}(t)-1\right]}{\cosh (2 t)-1} \\
& =1-\frac{2 \sum_{n=1}^{\infty} \frac{(2 n) !}{2^{2 n}(n !)^{4}} t^{2 n}}{\sum_{n=1}^{\infty} \frac{2^{2 n}}{(2 n) !} t^{2 n}}=1-\frac{2 \sum_{n=0}^{\infty} c_{n} t^{2 n}}{\sum_{n=0}^{\infty} d_{n} t^{2 n}}, \\
g\left(0^{+}\right) & =1-\frac{2 c_{0}}{d_{0}}=\frac{1}{2}, \\
\frac{1}{\pi(n+3 / 2)} & <\frac{c_{n}}{d_{n}}=\frac{\Gamma^{2}(n+3 / 2)}{\pi \Gamma^{2}(n+2)}<\frac{1}{\pi(n+1)},
\end{aligned}
$$




$$
g(\infty)=1-2 \lim _{n \rightarrow \infty} \frac{c_{n}}{d_{n}}=1
$$

Therefore, Lemma 8 follows easily from Lemma 4 and (2.13)-(2.16).

\section{MAIN RESULTS}

Theorem 1. Let $\lambda_{1}, \mu_{1}, \lambda_{2}, \mu_{2} \in[0,1 / 2]$. Then the double inequalities

$$
\begin{gathered}
H\left(a, b ; \lambda_{1}\right)<\alpha A(a, b)+(1-\alpha) T(a, b)<H\left(a, b ; \mu_{1}\right), \\
G\left(a, b ; \lambda_{2}\right)<\alpha A(a, b)+(1-\alpha) T(a, b)<G\left(a, b ; \mu_{2}\right)
\end{gathered}
$$

hold for all $\alpha \in[0,1]$ and $a, b>0$ with $a \neq b$ if and only if $\lambda_{1} \leq 1 / 2-\sqrt{1-\alpha} / 2$, $\mu_{1} \geq 1 / 2-\sqrt{1-\alpha} / 4, \lambda_{2} \leq 1 / 2-\sqrt{1-\alpha^{2}} / 2$ and $\mu_{2} \geq 1 / 2-\sqrt{2(1-\alpha)} / 4$.

Proof. Let $p, q \in[0,1 / 2]$. Without loss of generality, we assume that $b>a>0$ and $t=\log \sqrt{b / a}>0$ due to $A(a, b), T(a, b), H(a, b ; p)$ and $G(a, b ; q)$ are symmetric and homogeneous of degree one with respect to $a$ and $b$. From (1.1)-(1.5) one has

$$
\begin{aligned}
& A(a, b)=\sqrt{a b} \cosh (t), \\
& T(a, b)=\sqrt{a b} I_{0}(t), \\
& H(a, b ; p)=\sqrt{a b} \cosh (t)\left[1-(1-2 p)^{2} \frac{\sinh ^{2}(t)}{\cosh ^{2}(t)}\right], \\
& G(a, b ; q)=\sqrt{a b} \cosh (t) \sqrt{1-(1-2 q)^{2} \frac{\sinh ^{2}(t)}{\cosh ^{2}(t)}}, \\
& H(a, b ; p)-[\alpha A(a, b)+(1-\alpha) T(a, b)]=\frac{\sqrt{a b} \sinh ^{2}(t)}{\cosh (t)}\left[(1-\alpha) f(t)-(1-2 p)^{2}\right], \\
& G(a, b ; q)-[\alpha A(a, b)+(1-\alpha) T(a, b)] \\
& =\frac{\left.\sqrt{a b}\left[(1-\alpha)^{2}\left(\cosh ^{2}(t)-I_{0}^{2}(t)\right)+2 \alpha(1-\alpha)\left(\cosh ^{2}(t)-\cosh (t) I_{0}(t)\right)-(1-2 q)^{2}\right)\right]}{\cosh (t) \sqrt{1-(1-2 q)^{2} \frac{\sinh ^{2}(t)}{\cosh ^{2}(t)}}+\alpha \cosh (t)+(1-\alpha) I_{0}(t)} \\
& =\frac{\sqrt{a b} \sinh ^{2}(t)\left[2 \alpha(1-\alpha) f(t)+(1-\alpha)^{2} g(t)-(1-2 q)^{2}\right]}{\cosh (t) \sqrt{1-(1-2 q)^{2} \frac{\sinh ^{2}(t)}{\cosh ^{2}(t)}}+\alpha \cosh (t)+(1-\alpha) I_{0}(t)},
\end{aligned}
$$

where $f(t)$ and $g(t)$ are defined by Lemmas 7 and 8, respectively.

Therefore, Theorem 1 follows easily from (3.3) and (3.4) together with Lemmas 7 and 8 .

Remark 1. Let $\alpha=0$. Then inequalities (3.1) and (3.2) reduce to inequalities (1.8) and (1.9), respectively. 
Corollary 1. Let $\lambda_{1}=1 / 2-\sqrt{1-\alpha} / 2$ and $\mu_{1}=1 / 2-\sqrt{1-\alpha} / 4$. Then inequality (3.1) leads to

for all $t>0$.

$$
\frac{1}{\cosh (t)}<I_{0}(t)<\frac{3 \cosh (t)}{4}+\frac{1}{4 \cosh (t)}
$$

Corollary 2. Let $\lambda_{2}=1 / 2-\sqrt{1-\alpha^{2}} / 2$ and $\mu_{2}=1 / 2-\sqrt{2(1-\alpha)} / 4$. Then inequality (3.2)) leads to

$$
\frac{\sqrt{1-\alpha^{2}+\alpha^{2} \cosh ^{2}(t)}-\alpha \cosh (t)}{1-\alpha}<I_{0}(t)<\frac{\sqrt{\frac{1-\alpha}{2}+\frac{1+\alpha}{2} \cosh ^{2}(t)}-\alpha \cosh (t)}{1-\alpha}
$$

for all $\alpha \in(0,1)$ and $t>0$. In particular, if $\alpha=1 / 2$, then one has

$$
\sqrt{3+\cosh ^{2}(t)}-\cosh (t)<I_{0}(t)<\sqrt{1+3 \cosh ^{2}(t)}-\cosh (t)
$$

for all $t>0$.

\section{REFERENCES}

[1] M. Abramowitz and I. A. Stegun, Handbook of mathematical functions with formulas, graphs, and mathematical tables, ser. National Bureau of Standards Applied Mathematics Series. For sale by the Superintendent of Documents, U.S. Government Printing Office, Washington, D.C., 1964, vol. 55.

[2] M. Adil Khan, S. Begum, Y. Khurshid, and Y.-M. Chu, "Ostrowski type inequalities involving conformable fractional integrals," J. Inequal. Appl., pp. Paper No. 70, 14, 2018, doi: 10.1186/s13660-018-1664-4. [Online]. Available: https://doi.org/10.1186/s13660-018-1664-4

[3] G. D. Anderson, M. K. Vamanamurthy, and M. K. Vuorinen, Conformal invariants, inequalities, and quasiconformal maps, ser. Canadian Mathematical Society Series of Monographs and Advanced Texts. John Wiley \& Sons, Inc., New York, 1997, with 1 IBM-PC floppy disk (3.5 inch; HD), A Wiley-Interscience Publication.

[4] Z. Cai, J. Huang, and L. Huang, "Generalized Lyapunov-Razumikhin method for retarded differential inclusions: applications to discontinuous neural networks," Discrete Contin. Dyn. Syst. Ser. B, vol. 22, no. 9, pp. 3591-3614, 2017, doi: 10.3934/dcdsb.2017181. [Online]. Available: https://doi.org/10.3934/dcdsb.2017181

[5] Z. Cai, J. Huang, and L. Huang, "Periodic orbit analysis for the delayed Filippov system," Proc. Amer. Math. Soc., vol. 146, no. 11, pp. 4667-4682, 2018, doi: 10.1090/proc/13883. [Online]. Available: https://doi.org/10.1090/proc/13883

[6] Y.-M. Chu and M.-K. Wang, "Optimal Lehmer mean bounds for the Toader mean," Results Math., vol. 61, no. 3-4, pp. 223-229, 2012, doi: 10.1007/s00025-010-0090-9. [Online]. Available: https://doi.org/10.1007/s00025-010-0090-9

[7] L. Duan, X. Fang, and C. Huang, "Global exponential convergence in a delayed almost periodic Nicholson's blowflies model with discontinuous harvesting," Math. Methods Appl. Sci., vol. 41, no. 5, pp. 1954-1965, 2018, doi: 10.1002/mma.4722. [Online]. Available: https://doi.org/10.1002/mma.4722

[8] L. Duan, L. Huang, Z. Guo, and X. Fang, "Periodic attractor for reaction-diffusion high-order Hopfield neural networks with time-varying delays," Comput. Math. Appl., vol. 73, no. 2, pp. 233-245, 2017, doi: 10.1016/j.camwa.2016.11.010. [Online]. Available: https://doi.org/10.1016/j.camwa.2016.11.010 
[9] C. Huang, S. Guo, and L. Liu, "Boundedness on Morrey space for Toeplitz type operator associated to singular integral operator with variable Calderón-Zygmund kernel," J. Math. Inequal., vol. 8, no. 3, pp. 453-464, 2014, doi: 10.7153/jmi-08-33. [Online]. Available: https://doi.org/10.7153/jmi-08-33

[10] C. Huang and L. Liu, "Sharp function inequalities and boundness for Toeplitz type operator related to general fractional singular integral operator," Publ. Inst. Math. (Beograd) (N.S.), vol. 92(106), pp. 165-176, 2012, doi: 10.2298/PIM1206165H. [Online]. Available: https://doi.org/10.2298/PIM1206165H

[11] C. Huang and L. Liu, "Boundedness of multilinear singular integral operator with a non-smooth kernel and mean oscillation," Quaest. Math., vol. 40, no. 3, pp. 295-312, 2017, doi: 10.2989/16073606.2017.1287136. [Online]. Available: https://doi.org/10.2989/16073606.2017. 1287136

[12] C. Huang, Z. Yang, T. Yi, and X. Zou, "On the basins of attraction for a class of delay differential equations with non-monotone bistable nonlinearities," J. Differential Equations, vol. 256, no. 7, pp. 2101-2114, 2014, doi: 10.1016/j.jde.2013.12.015. [Online]. Available: https://doi.org/10.1016/j.jde.2013.12.015

[13] T.-R. Huang, B.-W. Han, X.-Y. Ma, and Y.-M. Chu, "Optimal bounds for the generalized Euler-Mascheroni constant,” J. Inequal. Appl., pp. Paper No. 118, 9, 2018, doi: 10.1186/s13660018-1711-1. [Online]. Available: https://doi.org/10.1186/s13660-018-1711-1

[14] T.-R. Huang, S.-Y. Tan, X.-Y. Ma, and Y.-M. Chu, "Monotonicity properties and bounds for the complete $p$-elliptic integrals," J. Inequal. Appl., pp. Paper No. 239, 11, 2018, doi: 10.1186/s13660-018-1828-2. [Online]. Available: https://doi.org/10.1186/s13660-018-1828-2

[15] M. A. Khan, Y.-M. Chu, A. Kashuri, R. Liko, and G. Ali, "Conformable fractional integrals versions of Hermite-Hadamard inequalities and their generalizations," J. Funct. Spaces, pp. Art. ID 6928 130, 9, 2018, doi: 10.1155/2018/6928130. [Online]. Available: https://doi.org/10.1155/2018/6928130

[16] M. A. Khan, Y. Chu, T. U. Khan, and J. Khan, "Some new inequalities of Hermite-Hadamard type for $s$-convex functions with applications," Open Math., vol. 15, no. 1, pp. 1414-1430, 2017, doi: 10.1515/math-2017-0121. [Online]. Available: https://doi.org/10.1515/math-2017-0121

[17] G. Pólya and G. Szegó, Problems and theorems in analysis. I, ser. Classics in Mathematics. Springer-Verlag, Berlin, 1998, series, integral calculus, theory of functions, Translated from the German by Dorothee Aeppli, Reprint of the 1978 English translation. [Online]. Available: https://doi.org/10.1007/978-3-642-61905-2. doi: 10.1007/978-3-642-61905-2

[18] F. Qi, X.-T. Shi, F.-F. Liu, and Z.-H. Yang, "A double inequality for an integral mean in terms of the exponential and logarithmic means," Period. Math. Hungar, vol. 75, no. 2, pp. 180-189, 2017, doi: 10.1007/s10998-016-0181-9. [Online]. Available: https://doi.org/10.1007/s10998-016-0181-9

[19] W.-M. Qian and Y.-M. Chu, "Sharp bounds for a special quasi-arithmetic mean in terms of arithmetic and geometric means with two parameters," J. Inequal. Appl., pp. Paper No. 274, 10, 2017, doi: 10.1186/s13660-017-1550-5. [Online]. Available: https://doi.org/10.1186/s13660-017-1550-5

[20] W.-M. Qian, X.-H. Zhang, and Y.-M. Chu, "Sharp bounds for the Toader-Qi mean in terms of harmonic and geometric means," J. Math. Inequal., vol. 11, no. 1, pp. 121-127, 2017, doi: 10.7153/jmi-11-11. [Online]. Available: https://doi.org/10.7153/jmi-11-11

[21] S.-L. Qiu, X.-Y. Ma, and Y.-M. Chu, "Sharp Landen transformation inequalities for hypergeometric functions, with applications," J. Math. Anal. Appl., vol. 474, no. 2, pp. 1306-1337, 2019, doi: 10.1016/j.jmaa.2019.02.018. [Online]. Available: https://doi.org/10.1016/ j.jmaa.2019.02.018 
[22] Y. Tan, C. Huang, B. Sun, and T. Wang, "Dynamics of a class of delayed reactiondiffusion systems with Neumann boundary condition," J. Math. Anal. Appl., vol. 458, no. 2, pp. 1115-1130, 2018, doi: 10.1016/j.jmaa.2017.09.045. [Online]. Available: https://doi.org/10.1016/j.jmaa.2017.09.045

[23] V. R. Thiruvenkatachar and T. S. Nanjundiah, "Inequalities concerning Bessel functions and orthogonal polynomials," Proc. Indian Acad. Sci., Sect. A., vol. 33, pp. 373-384, 1951.

[24] J. Wang, X. Chen, and L. Huang, "The number and stability of limit cycles for planar piecewise linear systems of node-saddle type," J. Math. Anal. Appl., vol. 469, no. 1, pp. 405-427, 2019, doi: 10.1016/j.jmaa.2018.09.024. [Online]. Available: https://doi.org/10.1016/j.jmaa.2018.09.024

[25] J.-L. Wang, W.-M. Qian, Z.-Y. He, and Y.-M. Chu, "On approximating the Toader mean by other bivariate means," J. Funct. Spaces, pp. Art. ID 6082 413, 7, 2019, doi: 10.1155/2019/6082413. [Online]. Available: https://doi.org/10.1155/2019/6082413

[26] M.-K. Wang, Y.-M. Chu, and W. Zhang, "Monotonicity and inequalities involving zero-balanced hypergeometric function," Math. Inequal. Appl., vol. 22, no. 2, pp. 601-617, 2019, doi: 10.7153/mia-2019-22-42. [Online]. Available: https://doi.org/10.7153/mia-2019-22-42

[27] W. Wang and Y. Chen, "Fast numerical valuation of options with jump under Merton's model," J. Comput. Appl. Math., vol. 318, pp. 79-92, 2017, doi: 10.1016/j.cam.2016.11.038. [Online]. Available: https://doi.org/10.1016/j.cam.2016.11.038

[28] H. Xi, L. Huang, Y. Qiao, H. Li, and C. Huang, "Permanence and partial extinction in a delayed three-species food chain model with stage structure and time-varying coefficients," J. Nonlinear Sci. Appl., vol. 10, no. 12, pp. 6177-6191, 2017, doi: 10.22436/jnsa.010.12.05. [Online]. Available: https://doi.org/10.22436/jnsa.010.12.05

[29] C. Yang and L. Huang, "New criteria on exponential synchronization and existence of periodic solutions of complex BAM networks with delays," J. Nonlinear Sci. Appl., vol. 10, no. 10, pp. 5464-5482, 2017, doi: 10.22436/jnsa.010.10.29. [Online]. Available: https://doi.org/10.22436/jnsa.010.10.29

[30] X. Yang, Q. Zhu, and C. Huang, "Generalized lag-synchronization of chaotic mix-delayed systems with uncertain parameters and unknown perturbations," Nonlinear Anal. Real World Appl., vol. 12, no. 1, pp. 93-105, 2011, doi: 10.1016/j.nonrwa.2010.05.037. [Online]. Available: https://doi.org/10.1016/j.nonrwa.2010.05.037

[31] Z.-H. Yang and Y.-M. Chu, "On approximating the modified Bessel function of the first kind and Toader-Qi mean,” J. Inequal. Appl., pp. Paper No. 40, 21, 2016, doi: 10.1186/s13660-016-0988-1. [Online]. Available: https://doi.org/10.1186/s13660-016-0988-1

[32] Z.-H. Yang and Y.-M. Chu, "A sharp lower bound for Toader-Qi mean with applications," $J$. Funct. Spaces, pp. Art. ID 4 165 601, 5, 2016, doi: 10.1155/2016/4165601. [Online]. Available: https://doi.org/10.1155/2016/4165601

[33] Z.-H. Yang, Y.-M. Chu, and Y.-Q. Song, "Sharp bounds for Toader-Qi mean in terms of logarithmic and identric means," Math. Inequal. Appl., vol. 19, no. 2, pp. 721-730, 2016, doi: 10.7153/mia-19-52. [Online]. Available: https://doi.org/10.7153/mia-19-52

[34] Z.-H. Yang, Y.-M. Chu, and M.-K. Wang, "Monotonicity criterion for the quotient of power series with applications," J. Math. Anal. Appl., vol. 428, no. 1, pp. 587-604, 2015, doi: 10.1016/j.jmaa.2015.03.043. [Online]. Available: https://doi.org/10.1016/j.jmaa.2015.03.043

[35] Z.-H. Yang, Y.-M. Chu, and W. Zhang, "Accurate approximations for the complete elliptic integral of the second kind," J. Math. Anal. Appl., vol. 438, no. 2, pp. 875-888, 2016, doi: 10.1016/j.jmaa.2016.02.035. [Online]. Available: https://doi.org/10.1016/j.jmaa.2016.02.035

[36] Z.-H. Yang, Y.-M. Chu, and W. Zhang, "High accuracy asymptotic bounds for the complete elliptic integral of the second kind," Appl. Math. Comput., vol. 348, pp. 552-564, 2019, doi: 10.1016/j.amc.2018.12.025. [Online]. Available: https://doi.org/10.1016/j.amc.2018.12.025 
[37] Z.-H. Yang, W.-M. Qian, and Y.-M. Chu, "Monotonicity properties and bounds involving the complete elliptic integrals of the first kind," Math. Inequal. Appl., vol. 21, no. 4, pp. 1185-1199, 2018, doi: 10.7153/mia-2018-21-82. [Online]. Available: https://doi.org/10.7153/ mia-2018-21-82

[38] Z.-H. Yang, W.-M. Qian, Y.-M. Chu, and W. Zhang, "Monotonicity rule for the quotient of two functions and its application," J. Inequal. Appl., pp. Paper No. 106, 13, 2017, doi: 10.1186/s13660-017-1383-2. [Online]. Available: https://doi.org/10.1186/s13660-017-1383-2

[39] Z.-H. Yang, W.-M. Qian, Y.-M. Chu, and W. Zhang, "On rational bounds for the gamma function,” J. Inequal. Appl., pp. Paper No. 210, 17, 2017, doi: 10.1186/s13660-017-1484-y. [Online]. Available: https://doi.org/10.1186/s13660-017-1484-y

[40] T.-H. Zhao, M.-K. Wang, W. Zhang, and Y.-M. Chu, "Quadratic transformation inequalities for Gaussian hypergeometric function,” J. Inequal. Appl., pp. Paper No. 251, 15, 2018, doi: 10.1186/s13660-018-1848-y. [Online]. Available: https://doi.org/10.1186/s13660-018-1848-y

\section{Authors' addresses}

Wei-Mao Qian

Wei-Mao Qian, School of Continuing Education, Huzhou Vocational \& Technical College, Huzhou 313000, Zhejiang, China

E-mail address: qwm661977@126.com

Wen Zhang

Wen Zhang, Friedman Brain Institute, Icahn School of Medicine at Mount Sinai, New York, NY 10029, United States

E-mail address: zhang.wen81@gmail.com

Yu-Ming Chu

Yu-Ming Chu (Corresponding author), Department of Mathematics, Huzhou University, Huzhou 313000, Zhejiang, China

E-mail address: chuyumingezjhu.edu.cn 